\section{Retinal layers thickness changes following epiretinal membrane surgery}

I Hecht, I Yeshurun, E Bartov, A Bar,

Z Burgansky-Eliash and A Achiron

\begin{abstract}
Purpose To evaluate the time course of changes in the thickness of retinal layers after epiretinal membrane (ERM) removal surgery. Methods A retrospective cohort study of patients following surgery for idiopathic ERM. We used new specialized image analysis software to create a thickness map of each retinal layer and analyzed changes during one year follow-up. Healthy fellow eyes were used as negative controls and the retina prior to surgery as positive control. Results Twenty-one patients were included with a mean age of $68 \pm 13$ years. Central macular thickness decreased steadily until 6 months after surgery (25\% decrease, $516 \pm 76$ to $386 \pm 73 \mu \mathrm{m}, P<0.001)$ with no further decrease between 6 and 12 months (386 \pm 73 to $390 \pm 73 \mu \mathrm{m}, P=0.291)$. The retinal nerve fiber layer (RNFL), and the ganglion cell and inner plexiform layer (GCIPL) were most affected $(57 \%, P<0.001$ and $27 \%, P=0.010$, respectively). The thickest region showed a more abrupt decrease of $21 \%$ at first followup $(504 \pm 61$ to $399 \pm 58 \mu \mathrm{m}, P=\mathbf{0 . 0 0 1})$ with subsequent decrements of about $3 \%$. Prior to surgery all retinal layers were thicker in study eyes compared with healthy control eyes $(6-63 \%$, all $P<0.05)$.

Conclusions Following ERM surgery, in the course of 6 months, the macula gradually becomes thinner after which a stable state is reached. All layers appear to be affected, with the RNFL and GCIPL impacted the most. Our results provide a unique view on how the thickness of different retinal layers changes

membrane (ILM). ${ }^{1}$ It can occur in proliferative retinopathies, inflammatory conditions, after blunt or penetrating trauma or as an idiopathic process in otherwise healthy eyes. ${ }^{1}$ Patients can be either asymptomatic or suffer from a myriad of symptoms including decreased visual acuity, metamorphopsia, and central photopsia. ${ }^{2}$

Intra-retinal changes can develop as a result of the ERM due to retinal traction and underlying anatomical disturbances. ${ }^{3}$ Following ERM removal surgery, further disturbances in retinal microstructure frequently develop such as alterations in foveal contour, inner retinal dimpling, and thinning or thickening of retinal areas. ${ }^{4-8}$

Several attempts have been made to ascertain the nature of retinal morphological changes following surgery, using various methods. $5,6,9$ Most focused on one specific aspect such as foveal contour or total retinal thickness. $5,6,9$ Given the time consuming nature of manually segmenting spectral-domain optical coherence tomography (SD-OCT) images, a description of the changes in individual layers following surgery was previously unavailable.

The aim of this study was to assess the effect of ERM peeling on retinal layer thickness in the course of 12 months following surgery. We used advanced image analysis software able to create a detailed thickness map of individual retinal layers from SD-OCT images automatically. A quantitative view of retinal layers following ERM surgery was acquired and their progression through time was analyzed.
\end{abstract} following ERM surgery.

Eye (2018) 32, 555-562; doi:10.1038/eye.2017.233; published online 10 November 2017

\author{
Introduction \\ Epiretinal membrane (ERM) is a fibrocellular \\ proliferation over the internal limiting
}

\section{Materials and methods}

We performed a retrospective observational study in the ophthalmic department of the Edith Wolfson Medical Center. The research followed the tenets of the Declaration of Helsinki.

Approval was obtained from the local Ethics Committee (approval number: 0013-15-WOMC).
Department of Ophthalmology, Edith Wolfson Medical Center and Sackler School of Medicine, Tel-Aviv University, Tel Aviv, Israel

Correspondence: I Hecht, Department of Ophthalmology, Edith Wolfson Medical Center and Sackler School of Medicine, 62 Halochamim Street, Holon 58100, Israel Tel: +972 3 5028706; Fax: +972 35028706 . E-mail: Idanhe@gmail.com

Received: 28 February 2017 Accepted in revised form: 15 September 2017 Published online: 10 November 2017 


\section{Patients}

We included patients diagnosed with symptomatic idiopathic ERM that were treated surgically at our institute during 2013-2016. Further inclusion criteria were: at least one high-quality SD-OCT scan in the month prior to surgery and at least two during the year following surgery. We excluded eyes with secondary ERM; defined as ERM occurring in a patient with any of the following diagnosis: proliferative diabetic retinopathy or severe non-proliferative diabetic retinopathy, history of intraocular injections, trauma, uveitis, intraocular surgery other than cataract extraction, venous occlusion, intraocular tumors, and retinal tears or detachment. Opposing eyes were included as the control group, given they had no evidence of ERM.

SD-OCT scans acquired prior to surgery and 1, 3, 6, and 12 months following surgery for both the study and the control eyes were included. Best-corrected visual acuity (BCVA) was measured at each appointment by a qualified nurse practitioner.

\section{Sample size}

We used the total retinal thickness (TRT) changes in a study of 117 patients with ERM following ERM peeling, ${ }^{5}$ and another study on 41 patients with idiopathic ERM following ERM and ILM peeling for sample calculations. ${ }^{6}$ Based on these studies, the mean TRT change was set as $94( \pm 54) \mu \mathrm{m}$. We found that a sample size of 4 patients was required to detect a difference with a significance level of 0.05 and a power of $80 \%$. Given our aims to measure individual layer thickness changes which could possibly be more subtle we decided to include at least triple this amount (ie, 12 patients) and therefore set a time frame of three years retrospectively. Calculations were performed using GLIMMPSE online software. ${ }^{10}$

\section{Surgical procedures}

All surgical procedures were performed by one of two experienced vitreoretinal surgeons (IY and EB). The surgery consisted of a 20-gauge pars plana vitrectomy (PPV) with use of triamcinolone acetate (Kenalog, BristolMyers Squibb Co, New York, NY, USA). The surgery was performed under general anesthesia in 18 cases $(86 \%)$ and retro-bulbar anesthesia in 3 cases (14\%). Six patients $(29 \%)$ underwent combined phacoemulsification with PPV. A posterior chamber intraocular lens was implanted in all of these cases. MembraneBlue-Dual (DORC International BV, Zuidland, Holland) was used to facilitate ERM peeling in 17 cases (81\%). Brilliant Blue G dye was used in 3 cases (14\%) and membrane blue in 1 case (5\%). The ILM was not routinely peeled, and was done so in only one case $(5 \%)$. At the end of the surgery partial air-fluid exchange was performed in 3 cases $(14 \%)$, the eye was filled with SF6 gas in 2 cases (10\%), and in 16 cases $(76 \%)$ no gas or air were left in the eye. A search for iatrogenic breaks was performed towards the end of the procedure with scleral indentation; areas suspicious for breaks were treated with cryotherapy in 2 cases $(10 \%)$. Peripheral endolaser was not routinely performed. Median duration of surgery was $50 \mathrm{~min}$ (Mean: 54, range, 27-91 min).

\section{Scan analysis}

All SD-OCT images were acquired using the Spectralis SD-OCT (Heidelberg Engineering, Heidelberg, Germany) system. Images of low quality were excluded (defined as images with a signal-to-noise ratio lower than 20 decibels as calculated by the Spectralis system ${ }^{11}$ ). SD-OCT analysis was performed using the Orion software (Voxeleron LLC, Pleasanton, CA, USA, software version 2.0.6043) using the same software version, workstation, and operating system for all analyses. ${ }^{12}$ The software has been previously used in studies assessing changes of retinal layers in the early course of ischemic optic neuropathy and was compared to manual segmentation. ${ }^{13,14}$ It showed good measurement repeatability and accuracy. ${ }^{13-15}$ The software was able to isolate the retinal nerve fiber layer (RNFL) and analyze it separately. However, it was unable to detect the demarcation between the ganglion cell and the inner plexiform layers and measured their thickness as one. For these reasons we chose to use the term 'GCIPL' for the ganglion cell and inner plexiform layers. Automated segmentation was reviewed manually for all scans insuring proper demarcation between layers. A thickness map of individual retinal layers was created from each scan and analyzed using the early treatment of diabetic retinopathy study (ETDRS) retinal grid. The central macula, inner annulus and outer annulus were analyzed individually as was the thickest region prior to surgery. Although manual cross checking was the primary method to insure accurate segmentation, we nonetheless tested the software for small inter-test variations which could potentially influence the results. We performed a measurement repeatability analysis by analyzing two random scans 5 times each. An example analysis and resulting thickness maps of one of our patients is presented in Figure 1.

\section{Statistical analysis}

Statistical analysis was performed using SPSS software (version 22.0, SPSS (IBM), Chicago, IL, USA). $P$ values $<0.05$ on a two sided test were considered statistically significant. We conducted $\chi^{2}$ tests for categorical 

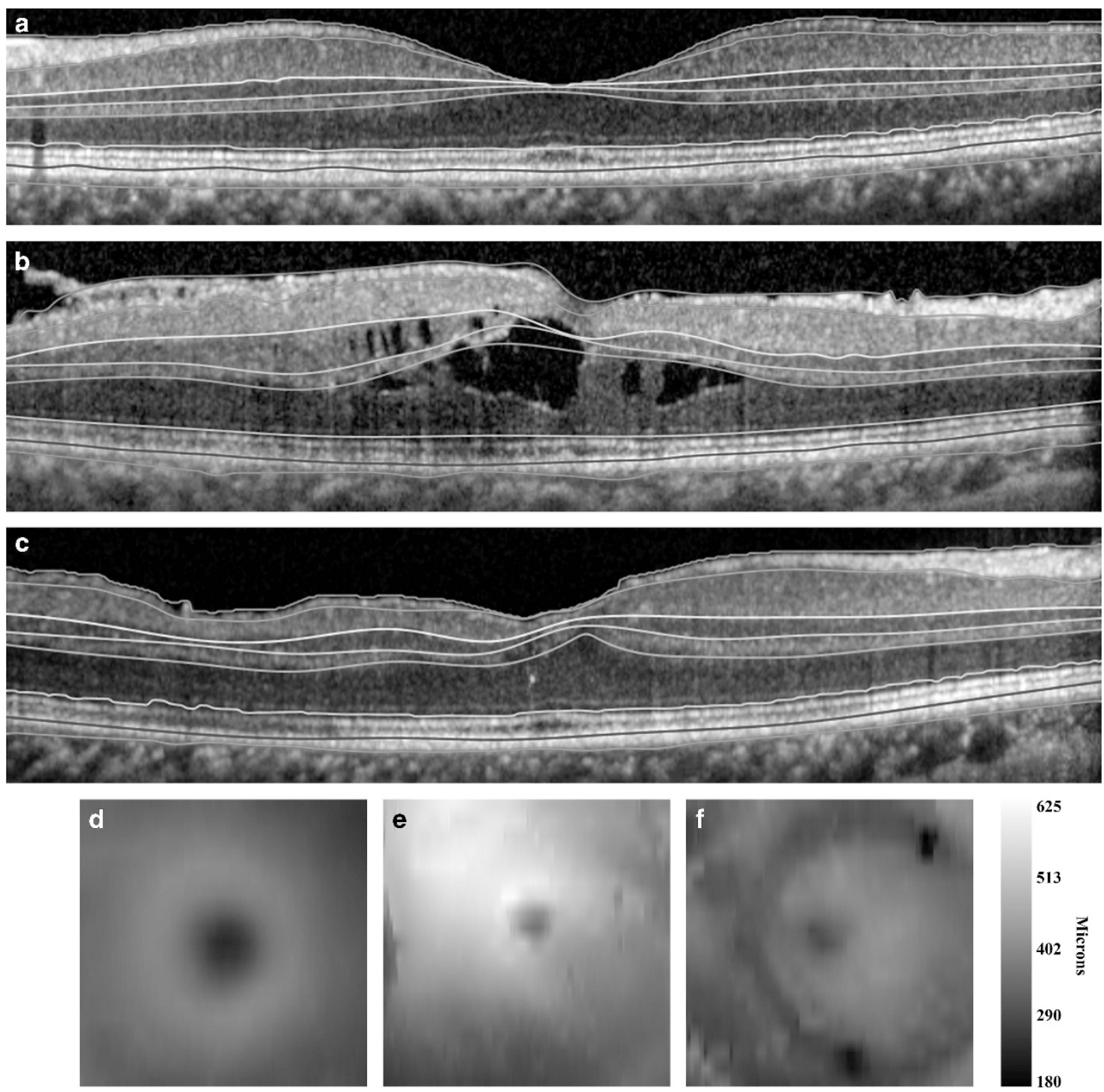

Figure 1 A representative retinal thickness analysis of one our patients, a 21-year-old male who suffered from a decrease in visual acuity in his right eye which progressed during the year prior to his admission. His medical and surgical histories were otherwise unremarkable. The left eye (control) is shown in a; the right eye is shown in b, lines represent borders between retinal layers generated automatically, notice the ERM causing traction and loss of the foveal pit. The same eye one year after surgery is shown in c, notice the thinning of the RNFL and GCIPL most prominent in the foveal region. (d) A thickness map of the TRT of the left (control) eye, the TRT of the right eye prior to surgery is shown in (e) and one year after surgery in f, notice the thickness of the temporal-superior region prior to surgery. ERM, epiretinal membrane; RNFL, retinal nerve fiber layer; GCIPL, ganglion cell and inner plexiform layer; TRT, total retinal thickness.

variables. Clinical parameters distributions were tested for normality by the Shapiro-Wilk test. Independent and paired $T$-tests were conducted for continuous variables with a normal distribution and Wilcoxon signed-rank test, and the Mann-Whitney $U$-test for variables with a non-normal distribution. For the measurement repeatability analysis variations were estimated by the difference between means and the intraclass correlation coefficient (ICC) using a two-way mixed model. In order to examine the differences between the excluded patients and the main cohort we tested for differences using the available data of the excluded group. Results are detailed in the online supplement (Supplementary Data). Unless otherwise specified, data are presented as mean \pm SD.

\section{Results}

Ninety-two patients underwent ERM surgery at our institute during 2013-2016, 42 met one of the exclusion criteria for non-idiopathic ERM and were therefore excluded. Twenty-one patients did not have a highquality SD-OCT scan in the month prior to surgery or at 


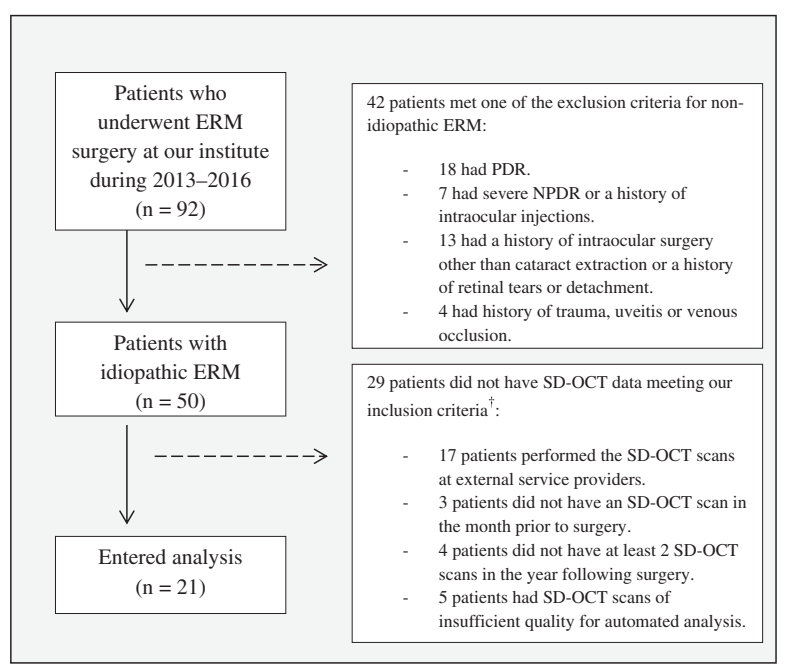

Figure 2 Flow diagram of the inclusion process. ${ }^{+} \mathrm{SD}-\mathrm{OCT}$ inclusion criteria were both a high-quality SD-OCT scan in the month prior to surgery and at least two high-quality SD-OCT scans in the year following surgery. Further details regarding the excluded patients can be found in the online supplement. ERM, epiretinal membrane; PDR, proliferative diabetic retinopathy; NPDR, non-proliferative diabetic retinopathy; SD-OCT, spectraldomain optical coherence tomography.

least two in the year following surgery and were also excluded. A flow diagram of the inclusion process is presented in Figure 2.

Twenty-one patients were included in the analysis. Age at the time of surgery averaged 68 years $( \pm 13$, range: 21-86), 9 were male (43\%). Symptom duration until surgery averaged 22 months ( \pm 23 , range: $3-84)$. Comorbid conditions included glaucoma (5/21, 24\%), mild non-proliferative diabetic retinopathy $(3 / 21,14 \%)$, status post cataract extraction $(5 / 21,24 \%)$, and dry eye syndrome $(1 / 21,5 \%)$. Cataract extraction was combined with the procedure in 6 cases $(29 \%)$. Following the procedure 11 patients were pseudophakic ( 6 following a combined surgery and 5 from previous cataract extraction) and 10 remained phakic.

All patients had between 3 and 5 SD-OCT scans (8 patients had 5 scans, 8 patients had 4 scans and 5 patients had 3 scans; median: 4). Scans were acquired prior to surgery (all patients), one months following surgery (14 patients), 3 months following surgery (17 patients), 6 months following surgery (16 patients) and 12 months following surgery (18 patients).

\section{Retinal layer thickness changes}

An overview of the retinal layer thickness changes following surgery is detailed in Figure 3 and in Animation 1 in the online supplement. Average TRT at the central macula was $516 \mu \mathrm{m}( \pm 76)$ prior to surgery and reduced to $390 \mu \mathrm{m}( \pm 73, P<0.001)$ at last follow-up. A similar decrease was noted at the inner annulus (462 to $351 \mu \mathrm{m}$, $P<0.001$ ) and the outer annulus (351 to $290 \mu \mathrm{m}$, $P<0.001)$.

The decrease in retinal thickness was most prominent during the first 6 months of follow-up (Figure 4). Examining the central macula, at 1 month following surgery a $10 \%$ decrease in TRT is seen $(516 \pm 76 \mu \mathrm{m}$ before to $464 \pm 84 \mu \mathrm{m}$ at 1 month after, $P=0.019)$, which progressed to $20 \%$ compared to preoperative values at 3 months $(464 \pm 84 \mu \mathrm{m}$ at 1 month to $413 \pm 78 \mu \mathrm{m}$ at 3 months, $P=0.080$ ) and to $25 \%$ compared to preoperative values at 6 months $(413 \pm 78 \mu \mathrm{m}$ at 3 months to $386 \pm 73 \mu \mathrm{m}$ at 6 months, $P=0.006)$. Between 6 and 12 months however there was no significant change $(386 \pm 73 \mu \mathrm{m}$ at 6 months to $390 \pm 73 \mu \mathrm{m}$ at 12 months, $P=0.291)$. A similar course of progression was also seen when analyzing the inner and outer annuluses as detailed in Figure 5.

In the segmentation analysis of the central macular area the RNFL and the ganglion cell and inner plexiform layer (GCIPL) were most affected with a 57\% (107 \pm 48 to $46 \pm 28 \mu \mathrm{m}, P<0.001)$ and $27 \%(112 \pm 30$ to $81 \pm 26 \mu \mathrm{m}$, $P=0.010)$ decrease in thickness at last follow-up respectively, while the outer plexiform layer (OPL) and photoreceptor layer (PR) were the only two layers to not change significantly (OPL: $41 \pm 6$ to $40 \pm 10 \mu \mathrm{m}, P=0.880$, PR: $71 \pm 8$ to $68 \pm 8 \mu \mathrm{m}, P=0.220)$ this is illustrated in Figure 4 . In the inner annulus again the RNFL was most affected with a $69 \%$ decrease in thickness at last follow-up ( $150 \pm 42$ to $47 \pm 14 \mu \mathrm{m}, P<0.001$ ) however the GCIPL, INL, and OPL did not change significantly. The outermost two layers (outer nuclear layer (ONL) and PR) both decreased by about $10 \%(P=0.001, P<0.001$ respectively) as illustrated in Figure 6. Finally, at the outer annulus the RNFL decreased the most $(52 \%, 103 \pm 26$ to $49 \pm 11 \mu \mathrm{m}, P<0.001)$ with most other layers only slightly decreasing in thickness or not at all. These results are illustrated in Figure 5.

The thickest region prior to surgery was located at the inner annulus in all subjects. The most common location was the inner temporal region seen in seven cases (33\%), followed by the inner nasal region in six cases $(29 \%)$, and lastly the inner superior and inferior regions with four cases each (19\%), these results are illustrated in Figure 6a. The progression of the thickest region was analyzed separately and illustrated in Figure $6 \mathrm{~b}$. The Thickest region shows a predominant decrease of the RNFL (75\%, $177 \pm 57$ to $45 \pm 9 \mu \mathrm{m}, P<0.001)$ and the two outer layers (ONL: $10 \%, 99 \pm 10$ to $89 \pm 10 \mu \mathrm{m}, P=0.020$ and PR: $15 \%$, $68 \pm 7$ to $58 \pm 4 \mu \mathrm{m}, \mathrm{P}=0.001)$. Contrary to the other retinal regions the decrease in thickness is more abrupt with a $21 \%$ significant decrease in total thickness at first follow-up (504 \pm 61 to $399 \pm 58 \mu \mathrm{m}, P=0.001)$ progressing with non-significant increments to $26 \%$ (399 \pm 58 to $374 \pm 39 \mu \mathrm{m}, P=0.106), 27 \%$ (374 \pm 39 to $366 \pm 38 \mu \mathrm{m}$, $P=0.234)$ and $29 \%(366 \pm 38$ to $356 \pm 37 \mu \mathrm{m}, \mathrm{P}=0.303)$ at 

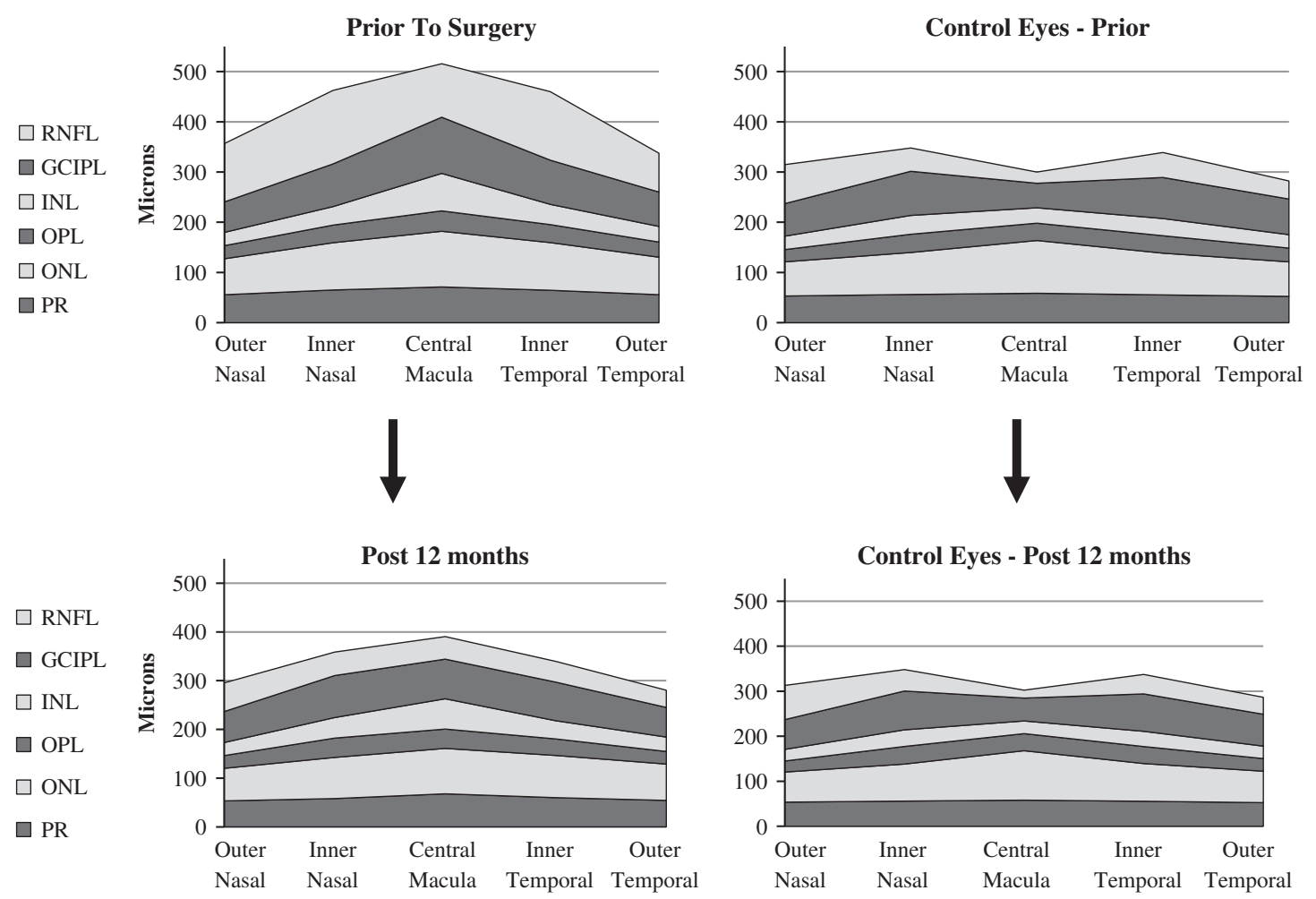

Figure 3 Overview of retinal layer progression following surgery. Note the decrease in thickness in the study eyes affecting mostly the inner layers. The control eyes show normal retinal architecture and did not change significantly during the follow-up period. RNFL, retinal nerve fiber layer; GCIPL, ganglion cell and inner plexiform layer; INL, inner nuclear layer; OPL, outer plexiform layer; ONL, outer nuclear layer; PR, photoreceptor layer.

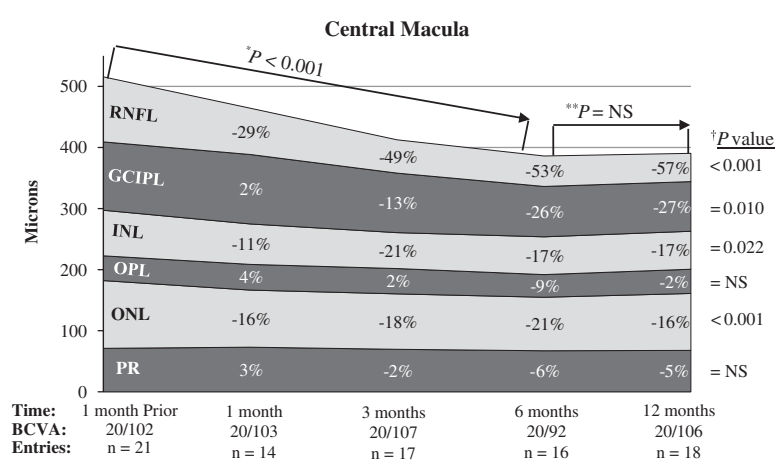

Figure 4 Central macular retinal layers before and after epiretinal membrane peeling. Percent (\%) quantities indicate the extent of thickness change relative to the thickness prior to surgery. Note the significant reduction in retinal thickness attained within 6 months followed by a relatively stable progression. The RNFL and GCIPL thickness decreased most dramatically while the OPL and PR were the only layers not to decrease significantly following surgery. *Total retinal thickness change before and 6 months following surgery. **Total retinal thickness change between 6 and 12 months following surgery. ${ }^{\dagger}$ Difference between the layer thickness prior to surgery and the end of follow-up. RNFL, retinal nerve fiber layer; GCIPL, ganglion cell and inner plexiform layer; INL, inner nuclear layer; OPL, outer plexiform layer; ONL, outer nuclear layer; PR, photoreceptor layer; BCVA, best-corrected visual acuity. subsequent follow-ups at 3, 6, and 12 months respectively (Figure 6b).

\section{Control eyes}

Analysis of the control eyes displayed normal retinal architecture with no significant change in any layer of any region during the follow-up period, achieving their main purpose as a negative control, and suggesting proper calibration of the system; these results are illustrated in Figure 3 and Animation 2 in the Supplementary Information.

Of the 21 opposing eyes, 4 also had an ERM (19\%). When comparing the healthy control eyes $(17 / 21$ opposing eyes) to the eyes with ERM prior to surgery all layers were significantly thicker in the ERM eyes. The RNFL, GCIPL, and the INL were most different at $63 \%$, $23 \%$, and $34 \%$ thicker, respectively (all $P<0.001$ ). The outer layers were also significantly thicker compared with the controls but to a lesser degree. The OPL, ONL, and PR were $6 \%, 8 \%$, and $14 \%$ thicker, respectively $(P=0.036$, $P=0.004$, and $P<0.001$, respectively).

In order to assess measurement repeatability, two SDOCT scans of two eyes were chosen at random, one from the study eyes and one from the control eyes. These were 

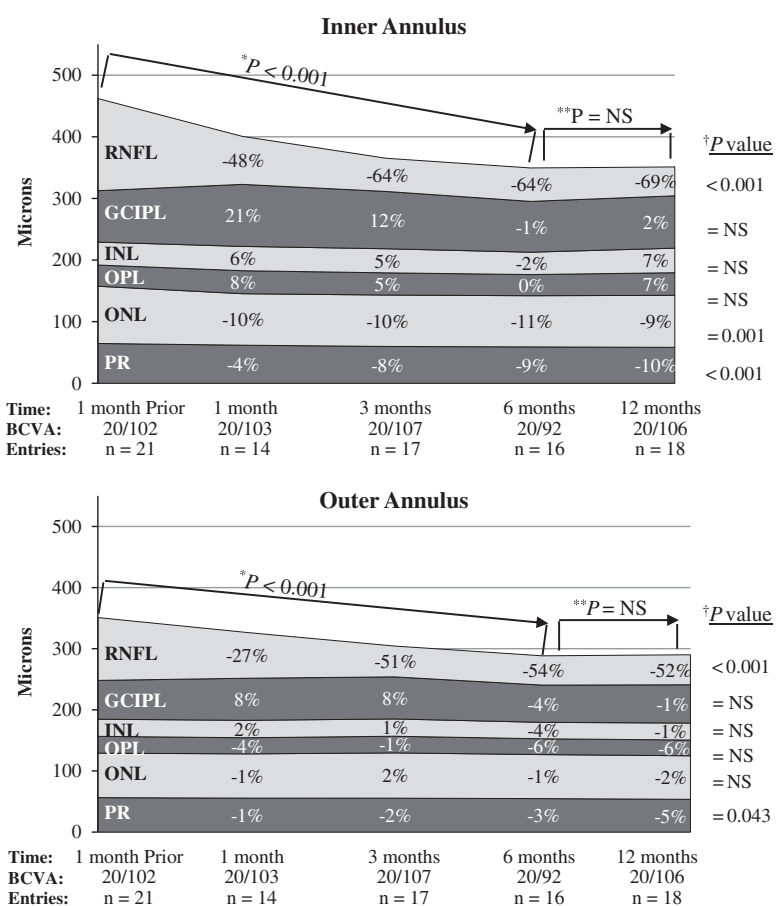

Figure 5 Inner and outer annulus progression. Percent (\%) quantities indicate the extent of thickness change relative to the thickness prior to surgery. ${ }^{*}$ Total retinal thickness change before and 6 months following surgery. ${ }^{* *}$ Total retinal thickness change between 6 and 12 months following surgery. ${ }^{+}$Difference between the layer thickness prior to surgery and the end of follow-up. RNFL, retinal nerve fiber layer; GCIPL, ganglion cell and inner plexiform layer; INL, inner nuclear layer; OPL, outer plexiform layer; ONL, outer nuclear layer; PR, photoreceptor layer; BCVA, best-corrected visual acuity.

analyzed repeatedly five times each. Differences between measurements were on average $0 \% \pm 0$ in the control eye (average ICC of 1.0 ) and $0.9 \% \pm 0.6$ in the study eye (average ICC of $0.999,95 \%$ confidence interval: 0.998-1.0). Detailed results are available in the online supplement (Supplementary Table 1). Manual cross checking of the automated segmentation was performed on all scans; minor corrections were performed in 3 scans of eyes with ERM and in none of the control eyes.

\section{Visual acuity}

Median visual acuity of the treated eyes was 20/93 (median: 0.669, interquartile range (IQR): 0.51-0.83 LogMAR) prior to surgery and did not change significantly until 6 months following surgery. Median visual acuity at 1 month after surgery was 20/99 (median: 0.698, IQR: 0.37-0.80 LogMAR, $P=0.611$ ), at 3 months 20/67 (median: 0.523, IQR: 0.41-0.80 LogMAR, $P=0.256$ ) and at 6 months there was slight non-significant improvement to 20/50 (median: 0.400, IQR: 0.33-0.69
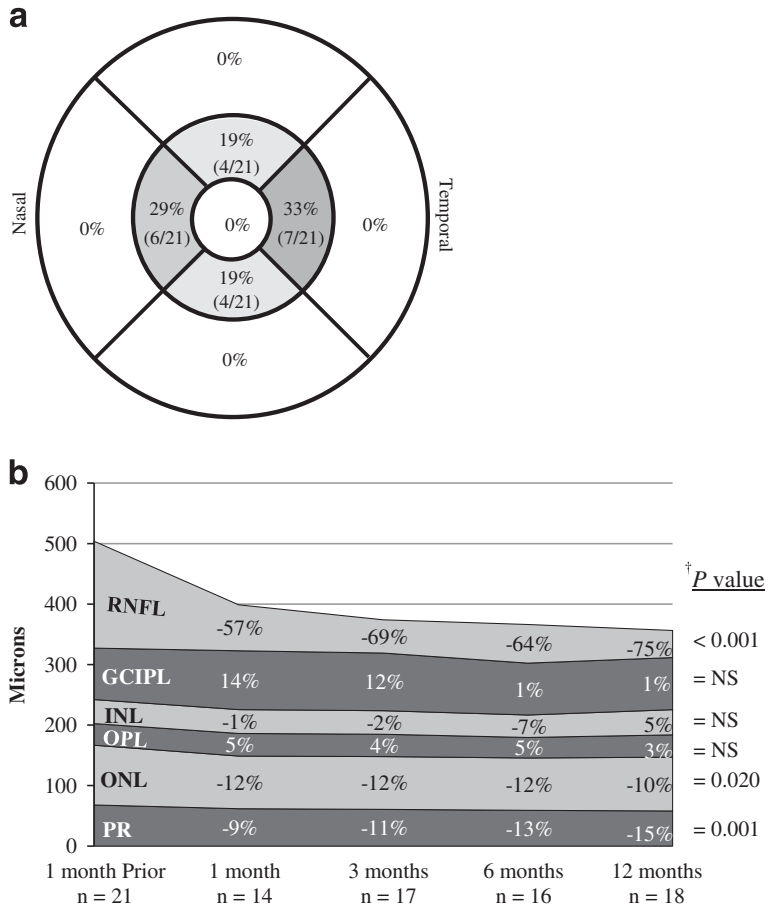

Figure 6 (a) Shows the location of the thickest region prior to surgery according to the ETDRS grid, gray-scale coloring, and percent quantities (\%) indicate the frequency of the thickest region occurring in that region. (b) Illustrates the relative thickness of each retinal layer during follow-up of the thickest region only, Percent (\%) quantities indicate the extent of thickness change relative to the thickness prior to surgery. ${ }^{t}$ Difference between the layer thickness prior to surgery and the end of follow-up. RNFL, retinal nerve fiber layer; GCIPL, ganglion cell, and inner plexiform layer; INL, inner nuclear layer; OPL, outer plexiform layer; ONL, outer nuclear layer; PR, photoreceptor layer; ETDRS, early treatment of diabetic retinopathy study.

LogMAR, $P=0.148)$. At 12 months following surgery visual acuity again worsened to 20/77 (median: 0.588, IQR: 0.47-0.84 $\log M A R, P=0.008)$.

Eyes that remained phakic after surgery $(n=10)$ improved from 20/81 (median: 0.610 IQR: 0.38-0.74 LogMAR) prior to surgery to 20/50 (median: 0.400 IQR: 0.22-0.59 LogMAR, $P=0.070) 6$ months following surgery. At 12 months visual acuity worsened to $20 / 78$ (median: 0.596 IQR: 0.46-0.77 LogMAR, $P=0.020$ ). During the year of follow-up, 6 patients developed cataract, of them 3 required cataract removal surgery. An illustration detailing the changes in visual acuity of phakic and pseudophakic eyes separately is available in the online supplement (Supplementary Figure 1).

Visual acuity was further tested for association with retinal thickness changes. Comparison of visual acuity in eyes with a mild decrease in macular thickness $(<104 \mu \mathrm{m}$, the median value) to those with a more profound decrease ( $>104 \mu \mathrm{m}$ ) showed small and statistically 
insignificant differences at 6 months (mild decrease, median: - 0.03 IQR: - 0.15-0.09 LogMAR; profound decrease, median: -0.08 IQR: - 0.33-0.18 LogMAR; $P=0.676)$ with similar results at 12 months post-operatively.

\section{Discussion}

In this study, we analyzed changes in retinal morphology due to idiopathic ERM and following the surgery for ERM removal. There are several important findings: (1) The majority of thickness decrease does not occur immediately but progresses steadily during 6 months. (2) After 6 months a stable state is reached with no further decrease. (3) The RNFL and GCIPL appear to be affected the most. (4) The outer layers also become thinner but to a lesser extent with an even smaller effect at the inner and outer annuluses. (5) Analysis of the thickest region shows a more abrupt course with the majority of the thickness decrease apparent at 1 month post-operatively. (6) All retinal layers of the ERM eyes prior to surgery were thicker than the control eyes, the inner layers by about $40 \%$ and the outer layers by about $10 \%$.

Wolf et $\mathrm{al}^{16}$ showed that when performing ILM peeling there is damage to the Müller cells (retinal glial cells) caused by the removal of their basement membranes and cell endfeet. The Müller cells span the entire thickness of the retina and are key mediators of retinal nerve cell protection. Damage to the Müller cells has been shown to accelerate retinal degeneration. ${ }^{17}$ The gradual thinning of retinal layers seen in our results, affecting the majority of layers but mostly the RNFL, could be explained by this process. Other researches have also implicated Müller cell damage with other post-operative phenomena such as late dimpling of the inner retinal surface. ${ }^{8}$

When examining the thickest region prior to surgery independently from the rest of the retina, the decrease in thickness is more abrupt, the majority of which occurs within one month after surgery (Figure 6). It is possible that this region is affected more directly by surgery and that the decrease in thickness is mainly due to a mechanical effect, while the rest of the retina is more prominently affected by secondary processes that progress more slowly.

Comparing the healthy control eyes to the eyes with ERM prior to surgery, all layers were significantly thicker in the ERM eyes, the inner layers by about $40 \%$ and the outer layers by $6-14 \%$ (all $P<0.05$ ). These results may imply that the force exerted by the ERM, causing traction and microstructural changes, may be affecting the inner layers further and mediated to the outer layers only to a lesser extent.

It has been shown by Romano et al ${ }^{18,19}$ that deep retinal changes can occur as a result of ERM and that these changes are correlated to functional outcomes. Although isolating specific intra-retinal phenomenon was not our current aim, a general effect on deep layer thickness is clearly seen. Further research into the ERM depth penetrance, deep intra-retinal changes and the corresponding visual outcomes could be an interesting avenue for future research.

Visual acuity was not significantly changed at most follow-up appointments in our cohort. Six months following surgery some improvement could be seen, but it as well did not reach statistical significance. It is possible that our study which was highly selective to include patients with high-quality, specifically timed SD-OCT scans, ended being underpowered to detect changes in visual acuity. Furthermore, close to half of patients remained phakic after surgery (10/21) and some developed cataract, a known late complication of the procedure. ${ }^{20}$ Trends and associations in other vision related symptoms such as metamorphopsia were not available since objective tests for these symptoms were not routinely performed and so these parameters were not analyzed. ${ }^{21}$

In this study, we used specialized software, recently developed by Voxeleron LLC (USA), to automatically analyze the SD-OCT volumes. Use of the software-enabled analysis not previously available due to the time consuming nature of retinal layer segmentation performed manually. The software has been used elsewhere, and showed good measurement repeatability and accuracy. ${ }^{13,15}$ We nevertheless conducted a measurement repeatability analyses which showed average differences of $0-0.9 \%$ (ICC 0.999-1.0) between measurements, suggesting good repeatability. Although not currently marketed for clinical use, the software could be employed in other projects involving precision measurement and bulk processing necessary for many types of research.

This study has a number of limitations. First, the study is based on retrospective analysis of a real-life cohort; therefore not all confounders can be accounted for or measured. Second, scan volume analysis was performed automatically by specialized software and demarcation between layers was reviewed manually; nevertheless the software used is still experimental in nature. Finally, we did not include patients who did not arrive to follow-up appointments and those with non-idiopathic ERM and our results cannot be generalized to those populations.

To conclude, our results suggest that following surgery for idiopathic ERM the retina gradually becomes thinner, during 6 months, after which a stable state is reached. All layers appear to be affected but mostly the RNFL and GCIPL. The thickest region was located exclusively at the inner annulus and shows a more abrupt course of progression. Our results shed light on retinal morphology after ERM removal surgery and may help to clarify retinal microstructural progression during this process. 


\section{Summary}

What was known before

- Intra-retinal changes can develop as a result of idiopathic epiretinal membranes.

- Surgery may lead to further disturbances in retinal microstructure.

- A description of these changes in individual layers before and following surgery was previously unavailable.

What this study adds

- Following surgery, in the course of 6 months, the macula gradually becomes thinner after which a stable state is reached.

- All retinal layers appear to be affected, with the two innermost layers affected the most.

- All retinal layers were thicker in eyes with epiretinal membranes prior to surgery compared with healthy control eyes.

\section{Conflict of interest}

The authors declare no conflict of interest.

\section{Acknowledgements}

The authors received no financial support for the research, authorship, and/or publication of this article. Use of the Orion software was provided free of charge by Voxelerone LLC.

\section{References}

1 Kampik A, Kenvon KR, Michels RG, Green WR, Delacruz ZC. Epiretinal and vitreous membranes comparative-study of 56 cases. Arch Ophthalmol 1981; 99: 1445-1454.

2 Bu SC, Kuijer R, Li XR, Hooymans JMM, Los LI. Idiopathic epiretinal membrane. Retina-J Ret Vit Dis 2014; 34: 2317-2335.

3 Romano MR, Cennamo G, Amoroso F, Montorio D, Castellani $\mathrm{C}$, Reibaldi $\mathrm{M}$ et al. Intraretinal changes in the presence of epiretinal traction. Graefes Arch Clin Exp Ophthalmol 2016; 255: 31-38.

4 Suh MH, Seo JM, Park KH, Yu HG. Associations between macular findings by optical coherence tomography and visual outcomes after epiretinal membrane removal. Am J Ophthalmol 2009; 147: 473-480.e473.

5 Kumagai K, Ogino N, Furukawa M, Hangai M, Kazama S, Nishigaki $\mathrm{S}$ et al. Retinal thickness after vitrectomy and internal limiting membrane peeling for macular hole and epiretinal membrane. Clin Ophthalmol 2012; 6: 679-688.

6 Mathews NR, Tarima S, Kim DG, Kim JE. Foveal contour changes following surgery for idiopathic epiretinal membrane. Invest Ophth Vis Sci 2014; 55: 7754-7760.
7 Niwa T, Terasaki $\mathrm{H}$, Kondo M, Piao $\mathrm{CH}$, Suzuki T, Miyake Y. Function and morphology of macula before and after removal of idiopathic epiretinal membrane. Invest Ophth Vis Sci 2003; 44: 1652-1656.

8 Pichi F, Lembo A, Morara M, Veronese C, Alkabes M, Nucci $\mathrm{P}$ et al. Early and late inner retinal changes after inner limiting membrane peeling. Int Ophthalmology 2013; 34: 437-446.

9 Hartmann KI, Schuster AK, Bartsch DU, Kim JS, Chhablani J, Freeman WR. Restoration of retinal layers after epiretinal membrane peeling. Retina-J Ret Vit Dis 2014; 34: 647-654.

10 Kreidler SM, Muller KE, Grunwald GK, Ringham BM, Coker-Dukowitz ZT, Sakhadeo UR et al. GLIMMPSE: online power computation for linear models with and without a baseline covariate. J Stat Softw 2013; 54: 1-26.

11 Balasubramanian M, Bowd C, Vizzeri G, Weinreb RN, Zangwill LM. Effect of image quality on tissue thickness measurements obtained with spectral domain-optical coherence tomography. Opt Express 2009; 17: 4019-4036.

12 Gronenschild EHBM, Habets P, Jacobs HIL, Mengelers R, Rozendaal N, van Os J et al. The effects of freesurfer version, workstation type, and macintosh operating system version on anatomical volume and cortical thickness measurements. PLoS One 2012; 7: e38234.

13 Oakley JD, Gabilondo I, Songster C, Russakoff D, Green A, Villoslada P. Assessing manual versus automated segmentation of the macula using optical coherence tomography. Invest Ophth Vis Sci 2014; 55: 4790-4790.

14 Keller J, Oakley JD, Russakoff DB, Andorrà-Inglés M, Villoslada P, Sánchez-Dalmau BF. Changes in macular layers in the early course of non-arteritic ischaemic optic neuropathy. Graefes Arch Clin Exp Ophthalmol 2015; 254: 561-567.

15 Tan J, Yang Y, Jiang H, Liu C, Deng ZH, Lam BL et al. The measurement repeatability using different partition methods of intraretinal tomographic thickness maps in healthy human subjects. Clin Ophthalmol 2016; 10: 2403-2415.

16 Wolf S, Schnurbusch U, Wiedemann P, Grosche J, Reichenbach A, Wolburg H. Peeling of the basal membrane in the human retina. Ophthalmology 2004; 111: 238-243.

17 Bringmann A, Reichenbach A. Role of Muller cells in retinal degenerations. Front Biosci 2001; 6: E77-E92.

18 Romano MR, Cennamo G, Cesarano I, Cardone D, Nicoletti G, Mastropasqua R et al. Changes of tangential traction after macular peeling: correlation between en-face analysis and macular sensitivity. Curr Eye Res 2017; 42: 780-788.

19 Romano MR, Cennamo G, Amoroso F, Montorio D, Castellani $\mathrm{C}$, Reibaldi $\mathrm{M}$ et al. Intraretinal changes in the presence of epiretinal traction. Graef Arch Clin Exp 2017; 255: 31-38.

20 Reilly G, Melamud A, Lipscomb P, Toussaint B. Surgical outcomes in patients with macular pucker and good preoperative visual acuity after vitrectomy with membrane peeling. Retina 2015; 35: 1817-1821.

21 Achiron A, Moisseiev E, Glick M, Yeshurun I, Bartov E, Burgansky Z. Quantification of metamorphopsia using the MacuFlow test before and after vitreoretinal surgery. Ophthalmic Res 2015; 54: 74-77. 